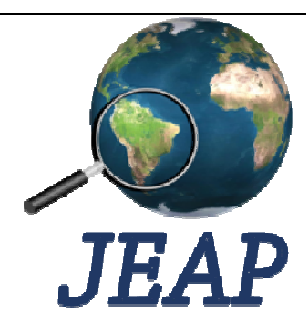

ISSN: 2525-815X

\section{Journal of Environmental} Analysis and Progress

Journal homepage: www.ufrpe.br/jeap

10.24221/jeap.2.4.2017.1670.549-554

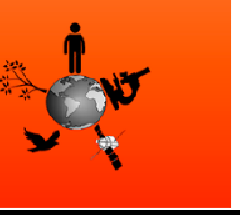

\title{
Variabilidade dos índices de umidade e aridez para as cidades de Crato e Paracurú no estado do Ceará
}

\section{Variability of moisture and aridity indices for the cities of Crato and Paracurú in the State of Ceará}

Verônica Gabriella de Oliveira ${ }^{\mathrm{a}}$, Irene Cristina Pereira Corrêa ${ }^{\mathrm{a}}$, José Ivaldo Barbosa de Brito ${ }^{\mathrm{a}}$, Adriana de Souza Costa ${ }^{a}$

a Universidade Federal de Campina Grande-UFCG, Rua Aprígio Veloso, n. 882, Campus Campina Grande-PB, Brasil. CEP: 58429-900. E-mail: gabriellamestrado2014@gmail.com; $\quad$ irenecristinacorrea1511@gmail.com; jose.ivaldo@ufcg.edu.br; adriana.asc24@gmail.com

A R T I C L E I N F O

Recebido 10 Ago 2017

Aceito 14 Set 2017

Publicado 27 out 2017

\begin{abstract}
A B S T R A C T
The interannual variability of NEB precipitation and its relation to the ENSO phenomenon has been the goal of many studies. However, most of the time no climate analysis is performed, but only the variability of the variables precipitation and air temperature, or even just precipitation. The objective of this study was to analyze the interannual variability of the aridity index of UNEP - Ia (UNEP) and effective humidity (Im) through the harmonic analysis for the cities of Crato and Paracurú in Ceará. The data manipulated were the mean monthly air and monthly and annual total precipitation temperatures for the period 1951 to 2014, which corresponds to 64 years of data. For the estimation of the indices the water balance was used. It was concluded that the municipalities have as main forces responsible for the variability of the climate the El Niño and La Niña phenomena.
\end{abstract}

Keywords: $\mathrm{I}_{\mathrm{a}}(\mathrm{UNEP}), \mathrm{I}_{\mathrm{m}}$, harmonic analysis.

\begin{abstract}
R E S U M O
A variabilidade interanual da precipitação do NEB e sua relação com o fenômeno ENOS, tem sido o objetivo de muitos estudos. Entretanto, na maioria das vezes nenhuma análise climática é realizada, mas apenas a variabilidade das variáveis precipitação e temperatura do ar, ou mesmo só a precipitação. Neste estudo, o objetivo foi analisar a variabilidade interanual do índice de aridez da UNEP $\mathrm{I}_{\mathrm{a}(\mathrm{UNEP})}$ e efetivo de umidade $\left(\mathrm{I}_{\mathrm{m}}\right)$ através da análise harmônica para as cidades de Crato e Paracurú no Ceará. Os dados manipulados foram os de temperaturas do ar médias mensais e de precipitação totais mensais e anuais para o período de 1951 a 2014, o que corresponde a sessenta e quatro anos de dados. Para a estimativa dos índices usou-se o balanço hídrico. Foi concluído que os municípios possuem como suas principais forçantes responsáveis pela variabilidade do clima os fenômenos El Niño e La Niña.

Palavras-Chave: $\mathrm{I}_{\mathrm{a}(\mathrm{UNEP})}, \mathrm{I}_{\mathrm{m}}$, análise harmônica.
\end{abstract}

\section{Introdução}

Os fenômenos climatológicos são considerados como periódicos, visto que se repetem a intervalos regulares de tempo e espaço. A precipitação ocorre de forma periódica e compõe um dos fatores mais importantes na produção agrícola. Considerando os componentes climáticos, a precipitação é um dos fatores que mais afetam a produção agrícola, devido a seu caráter aleatório.

O clima do Nordeste é complexo, apresentando grande variabilidade espaço- 
temporal da precipitação, elevada taxa de evaporação e temperatura média elevada, variando entre $24^{\circ} \mathrm{C}$ e $28^{\circ} \mathrm{C}$. O máximo de precipitação no norte do Nordeste, principalmente nos estados do Maranhão, Ceará, oeste do Rio Grande do Norte e interior da Paraíba e Pernambuco, ocorre no período de fevereiro a maio e deve-se ao deslocamento da Zona de Convergência Intertropical (ZCIT) para latitudes mais ao sul, a qual influencia diretamente na qualidade do período chuvoso da região (Uvo, 1989).

Conforme a classificação de Köppen, o Ceará possui o clima do tipo As Bs'h, clima quente e semiárido com chuvas de outono e temperatura média sempre superior a $18^{\circ} \mathrm{C}$ (Fundação Cearense de Meteorologia e Recursos hídricos - FUNCEME, 2016).

Thornthwaite (1948) elaborou os índices de umidade, aridez e efetivo de umidade objetivando a classificação climática. Em seguida, Thornthwaite \& Mather (1955) propuseram um balanço hídrico tendo com variáveis de saída a evapotranspiração potencial total anual, déficit anual de umidade na atmosfera, excedente anual de água no solo, entre outras. Além disso, estes autores indicaram que o Índice de Aridez $\left(I_{a}\right)$ é a razão entre o déficit anual de umidade e a evapotranspiração potencial, o Índice de Umidade ( $\left.\mathrm{I}_{u}\right)$ é o excedente anual de água no solo dividido pela evapotranspiração potencial, e o Índice Efetivo de Umidade $\left(I_{m}\right)$ é o $\left(I_{u}\right)$ multiplicado por 100 menos 0,6 vezes $\left(I_{a}\right)$, multiplicado por 100 .

Budyko (1958) propôs um índice climático simples, capaz de classificar o clima de uma determinada área, como a razão da evapotranspiração potencial total média anual e a precipitação total média anual. Este índice, posteriormente, foi denominado índice de aridez de Budyko $\left(\mathrm{I}_{\mathrm{ab}}\right)$.
Hare (1972) modificou o $\mathrm{I}_{\mathrm{ab}}$, invertendo a razão, ou seja, o novo índice de aridez é a razão entre a precipitação total média anual e a evapotranspiração, ou seja, é um índice de aridez inverso de Budyko (1958). Este índice tem sido utilizado e indicado pelo Programa das Nações Unidas para o Meio Ambiente (UNEP - United Nations Environment Programme) na classificação de terras susceptíveis aos processos de desertificação. Por este motivo, ele tem sido denominado de índice de aridez do UNEP.

Neste contexto, o objetivo deste estudo foi avaliar a variabilidade do índice de efetivo de umidade e aridez através da análise harmônica para as cidades de Crato e Paracurú no Ceará.

\section{Material e Métodos}

Área de estudo

A área de estudo são duas cidades do estado do Ceará, localizadas um pouco abaixo da linha do Equador, em uma posição nitidamente tropical, entre $2^{\circ} 46^{\prime} 30^{\prime \prime}$ e $7^{\circ} 52^{\prime} 15^{\prime \prime}$ de latitude Sul e $37^{\circ} 14^{\prime} 54^{\prime \prime}$ e $41^{\circ} 24^{\prime} 45^{\prime \prime}$ de longitude Oeste, especificamente na região Nordeste do Brasil. Estão limitadas, ao norte, com o Oceano Atlântico, ao sul, com o estado de Pernambuco, a leste com os estados do Rio Grande do Norte e Paraíba e, a oeste, com o estado do Piauí (Figura 1a). A área total do Estado do Ceará é de $148.825 .6 \mathrm{~km}^{2}$, o que equivale, aproximadamente, a $9,6 \%$ da área pertencente à região Nordeste e $1,8 \%$ da área do Brasil, com população de 8.842.791 habitantes (IBGE, 2014). Os dois postos escolhidos, espacialmente opostos, Paracurú localizado no lado norte e Crato no lado sul, do estado do Ceará, podem ser localizados no mapa da Figura 1b.

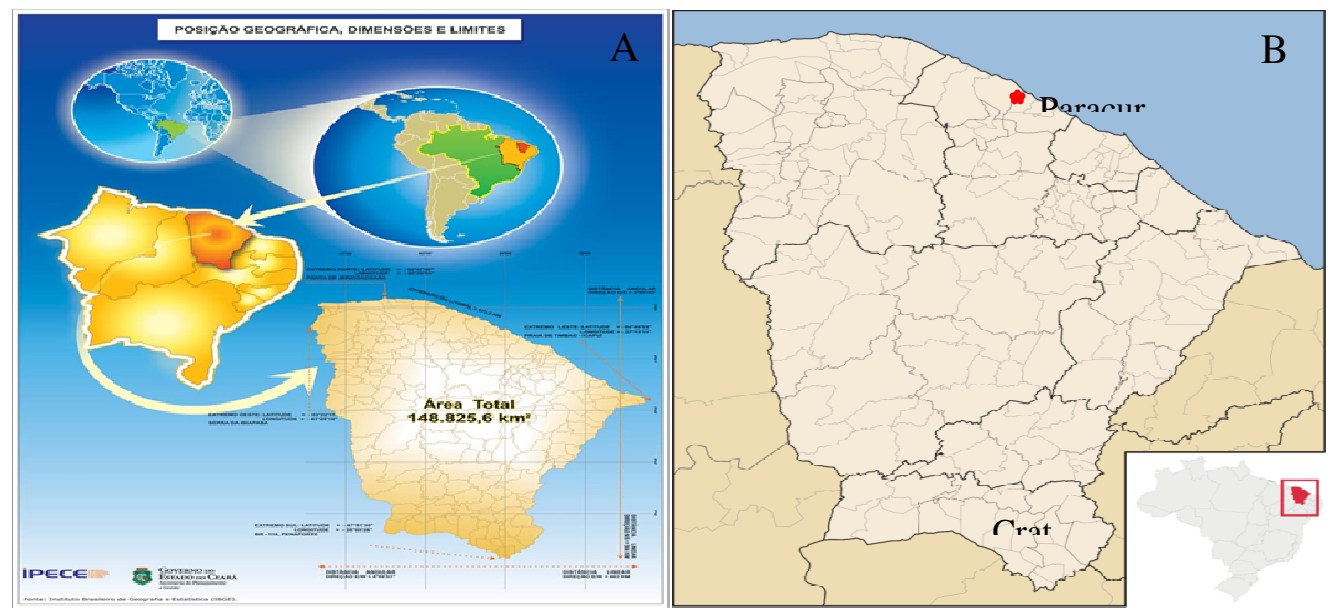

Figura 1. Mapas mostrando a localização da área de estudo. A. Localização da área de estudo; B. Localização dos dois postos pluviométricos. Fonte: Instituto de Pesquisa e Estratégia Econômica do Ceará (IPECE) (2007). Fonte: Google Maps, adaptado pelos autores. 


\section{Dados}

Os dados utilizados neste estudo foram as médias mensais de temperaturas do ar $\left({ }^{\circ} \mathrm{C}\right)$ do período de 1951 a 2014, dos dois municípios do Estado do Ceará selecionados nesta pesquisa, estes dados foram estimados através do software, Estima_T (http://www.dca.ufcg.edu.br / download lestimat.htm), que faz estimativas de temperaturas do ar na Região Nordeste do Brasil. Este software divide a região nordeste em três áreas: 1Maranhão e Piauí; 2- Ceará, Rio Grande do Norte, Paraíba e Pernambuco e 3- Alagoas; Sergipe e Bahia. Segundo Cavalcanti \& Silva (1994), Cavalcanti et al. (2006) e Silva et al. (2006), para cada uma das regiões foram determinados os coeficientes da função quadrática para as temperaturas média, máxima e mínima mensal em função das coordenadas locais: longitude, latitude e altitude. Para a obtenção das temperaturas média, máxima e mínima, mês a mês, para cada localidade, foram utilizadas as anomalias de TSM do Oceano Atlântico Tropical.

Foram utilizados dados de Precipitação para as localidades, fornecidos pela Fundação Cearense de Meteorologia e Recursos Hídricos do estado do Ceará (FUNCEME) e do Instituto Nacional de Meteorologia (INMET), para séries históricas que compreendem o período de 1951 a 2014 (63 anos).

\section{Índices}

O índice de aridez do UNEP (Ia(UNEP)) é calculado dividindo a precipitação total anual da localidade pela evapotranspiração potencial total anual. A estimativa da evapotranspiração pode ser realizada por diversos métodos, porém neste trabalho foi usada a estimada pelo método de Thornthwaite (1948).

Os índices de aridez $\left(\mathrm{I}_{\mathrm{a}}\right)$ e efetivo de umidade $\left(I_{m}\right)$ de Thornthwaite (1948) para as duas localidades selecionadas foram obtidos através do balanço hídrico seriado conforme o modelo proposto por Thornthwaite \& Mather (1955) e desenvolvido em planilha Excel por Rolim \& Sentelhas (1998). O balanço hídrico foi executado para as duas localidades e os 63 anos de dados. A capacidade de água disponível (CAD) utilizada foi de $100 \mathrm{~mm}$. O balanço hídrico tem como variáveis de saída, a evapotranspiração potencial total anual, a deficiência hídrica total anual da atmosfera e o excedente de água no solo total anual. Com estas três variáveis foi possível calcular $\mathrm{I}_{\mathrm{a}}$ e $\mathrm{I}_{\mathrm{b}}$. Além dessas variáveis, o balanço hídrico ainda tem como variável de saída, o conteúdo médio anual de umidade do solo e a evapotranspiração real total anual.

\section{Análise harmônica}

A análise harmônica é um dos métodos de estudo dos fenômenos periódicos ou quase periódicos (aquele em que o valor do elemento estudado não se reproduz exatamente em certos intervalos de tempo, como se a um processo estritamente periódico, se superpusessem influencias modificadoras, de caráter aleatório). Desses métodos, é a Análise harmônica, sem dúvida, o mais bem sistematizado, sendo de aplicação obrigatória quando o período fundamental é conhecido e o processo não se reduz, sabidamente, a uma onda senoidal simples, tal como ocorre com o curso anual ou diurno de um elemento climático de uma localidade.

Seguindo o conceito de análise harmônica é oportuno descreve-la na forma matemática. Portanto, a partir de uma série temporal de dados de uma variável climatológica $\mathrm{f}(\mathrm{t})$, observada em um intervalo de tempo $\Delta \mathrm{t}$, durante um período t, produzindo um número total de observações $\mathrm{N}$ igual a $t / \Delta t$. A variável $f(t)$ pode ser representada por uma série trigonométrica dada pela Equação 1:

$$
\mathrm{F}\left(\mathrm{t}_{\mathrm{i}}\right)=\mathrm{a}_{0}+\sum_{K=1}^{2}\left(a_{\mathrm{h}} \cos \left(\mathrm{K} \omega t_{i}\right)+\mathrm{b}_{\mathrm{k}} \operatorname{sen}\left(\omega t_{i}\right)\right) \mathrm{Eq} \cdot(1)
$$

No caso especifico, $\mathrm{f}\left(\mathrm{t}_{\mathrm{i}}\right)$ é o índice de umidade efetivo $\left(\mathrm{I}_{\mathrm{m}}\right)$ e o índice de aridez da Unep ( $\left.\mathrm{I}_{\mathrm{a}(\mathrm{UNEP})}\right)$ estimado ano a ano de 1951 a 2014 para os dois municípios do estado do Ceará.

\section{Resultados}

De acordo com Thornthwaite \& Mather (1955), os índices de aridez e efetivo de umidade servem de base para a classificação climática. É mostrado a variabilidade anual dos $\mathrm{I}_{\mathrm{a}(\mathrm{UNEP})}$ e do $\mathrm{I}_{\mathrm{m}}$ para a cidade do Crato. Valores acima de 1,00 indicam menor grau de aridez, como podemos notar nos anos de 1963, 1974, 1977, 1985 e 2011, anos que ocorreram o fenômeno da la niña e o dipolo de anomalias de TSM do atlântico tropical foi negativo. $\mathrm{O} \mathrm{I}_{\mathrm{m}}$ quanto menor $\mathrm{o}$ valor do mesmo, maior é a sua deficiência hídrica na atmosfera, como mostrado nos anos de 1958, 1990, 1993, 1997 e 2012 (Figura 2). Para verificar os aspectos da variabilidade desses índices utilizou-se a análise harmônica, onde é possível confirmar, na Figura 3, que os índices $I_{a(U N E P)}$ e $I_{m}$ apresentam resultados de ciclos estatisticamente significativos, sendo possível verificar que os ciclos de maiores frequência da análise harmônica têm variabilidade interanual, ou seja, maior periodicidade nesta escala ao longo do tempo, com frequência aproximada de 63 anos $(0,07 ; 4)$, 10 anos( $(0,10), 8$ anos $(0,09), 5-4$ anos $(; 0,06), 3-2$ anos $(0,06-0,07)$. Esses resultados representam os 
valores de maiores picos, visto que foram encontrados valores de periodicidade com significância superiores a 90\% (p_valor $<0,1$ ). Considerando a analise harmônica dos ciclos de maior frequência, é possível constatar que os harmônicos possuem variabilidade interanual, isto é, maior periodicidade nesta escala ao longo do tempo, estando relacionada com o El NiñoOscilação Sul (ENOS) e a ZCIT. Em relação a esse período de observação, percebe-se que foi verificada maior ocorrência do fenômeno da La Niña fazendo, desta forma, com que fossem originados esses picos elevados. A respeito desses declínios com frequência de 9 a 25 anos, com valores de amplitude de 0,01 , em ambos os anos, estes podem estar relacionados com os fenômenos El Niño, portanto, é notório que estes índices apresentam a mesma variabilidade interanual e interdecenal.

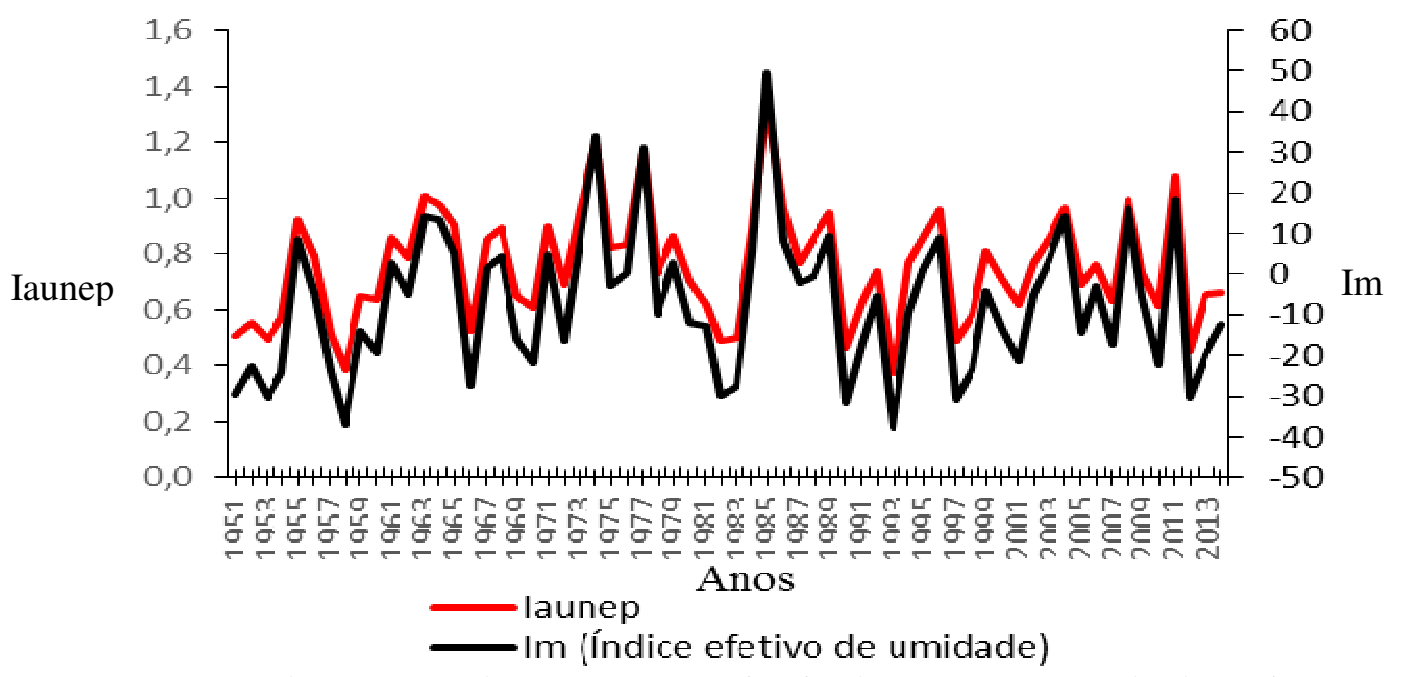

Figura 2. Comportamento do $\mathrm{I}_{\mathrm{a}(\mathrm{UNEP})}$ e do $\mathrm{I}_{\mathrm{m}}$ para o município de Crato no período de 1951 a 2014. Fonte: Corrêa, I.C.P. (2016).

A
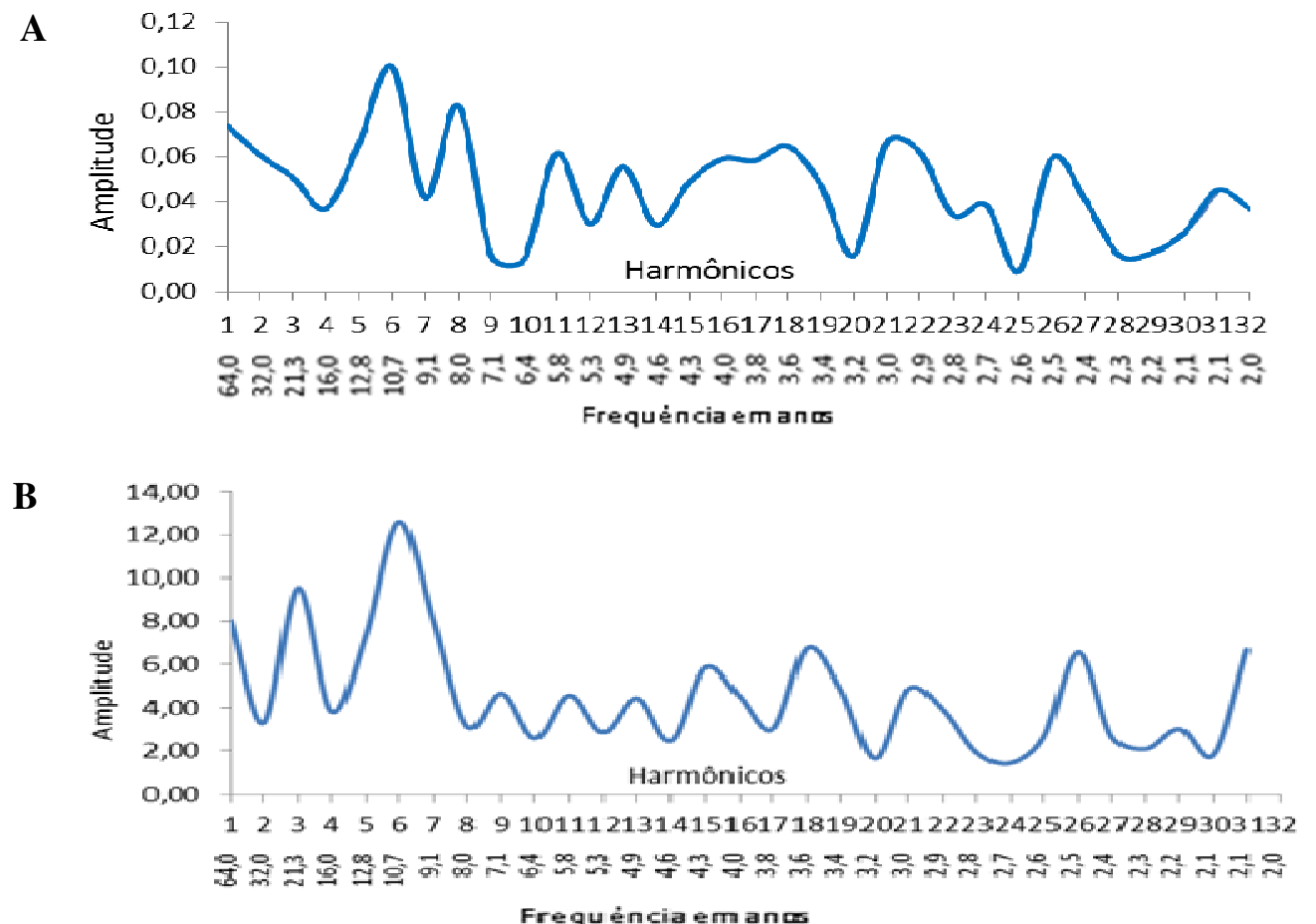

Figura 3. Análise Harmônica do A- $\mathrm{I}_{\mathrm{a}(\mathrm{UNEP})}$ e B- $\mathrm{I}_{\mathrm{m}}$ para o município de Crato. Fonte: Corrêa, I.C.P. (2016).

Na Figura 4 é mostrada a variabilidade anual dos $\mathrm{I}_{\mathrm{a}(\mathrm{UNEP})}$ e do $\mathrm{I}_{\mathrm{m}}$ para a cidade de Paracurú. Verificou-se que os anos com maior aridez foram 1951, 1958, 1967, 1990 e 2012, onde as ocorrências variam entre forte/moderado do fenômeno El niño e também a anomalia de TSM 
positiva do atlântico tropical. $\mathrm{O} \mathrm{I}_{\mathrm{m}}$ segue a mesma tendência que o $\mathrm{I}_{\mathrm{a}(\mathrm{UNEP})}$, verificou-se que os anos com maior "abundancia" hídrica atmosférica ocorrem em anos de TSM negativa e La niña (1964 e 1974).

Dos 63 anos analisados (1951 a 2014), usando o $\mathrm{I}_{\mathrm{a}(\mathrm{UNEP}) \text {, é possível quantificado as classes }}$ de anos de formas diferentes, assim como também com a utilização do $I_{m}$ é possível classificar os intervalos de anos de formas diferentes, isto é, de acordo com os fenômenos meteorológicos e acontecimentos climáticos, pode-se caracterizar o clima de acordo com os eventos ocorridos naqueles anos.

Na Figura 5ab verificou-se, após análise harmônica, ciclos estatisticamente significativos iguais para essas duas variáveis de 32 anos (15), 10 anos (15), 8 anos (11), 5-4 anos (10-12) e 3-2 anos (4-10). Estes valores compõem as classes que são de maior pico, os ciclos de maiores frequências da análise harmônica tem variabilidade interanual, ou seja, maior periodicidade nesta escala ao longo do tempo, com frequência aproximada de seis anos, estando relacionada com o El Niño-Oscilação Sul (ENOS) e a ZCIT.

Ainda sobre o comportamento dessas variáveis para Paracurú, ao fazer uma comparação dos índices de aridez $\mathrm{I}_{\text {a(UNEP) }}$ com o efetivo de umidade é possível observar que, as mesmas, se comportam de formas idênticas, diferentemente do que é observado no comportamento dessas variáveis para o Crato. Os resultados mostraram que o sinal da chuva está modulado por sistemas e/ou mecanismos atmosféricos atuantes em diferentes escalas temporais.

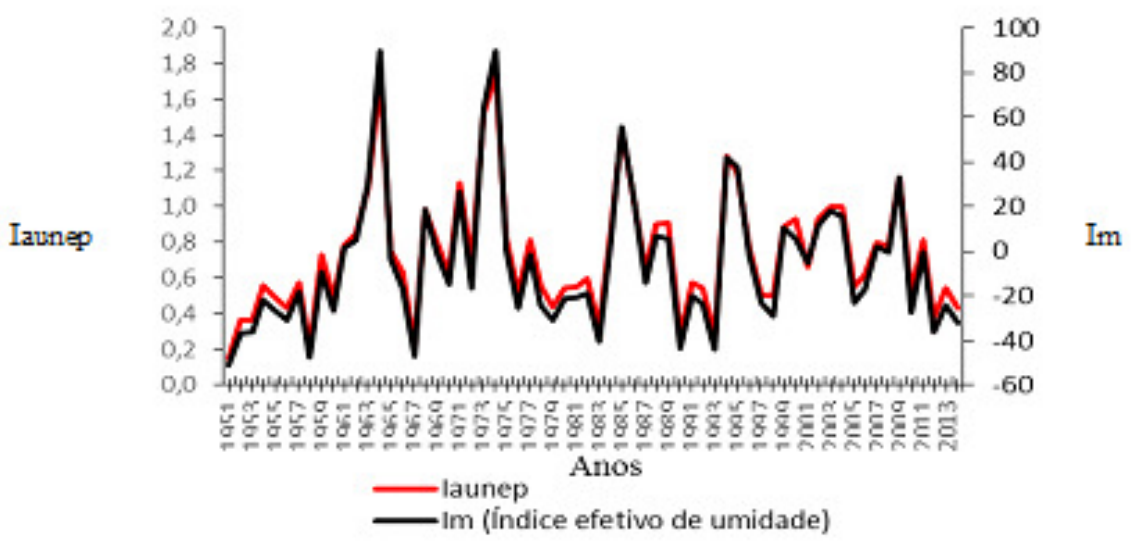

Figura 4. Comportamento do $\mathrm{I}_{\mathrm{a}(\mathrm{UNEP})}$ e do $\mathrm{I}_{\mathrm{m}}$ para o município de Paracurú no período de 1951 a 2014. Fonte: Corrêa, I. C. P. (2016).

A

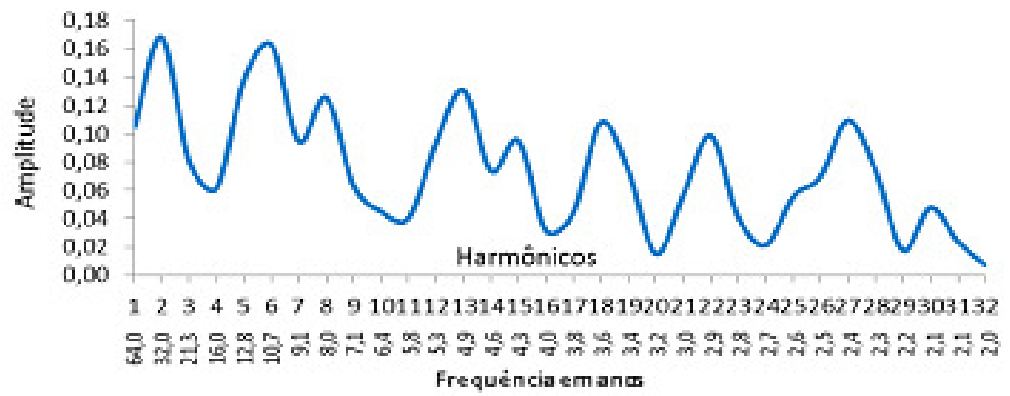

B

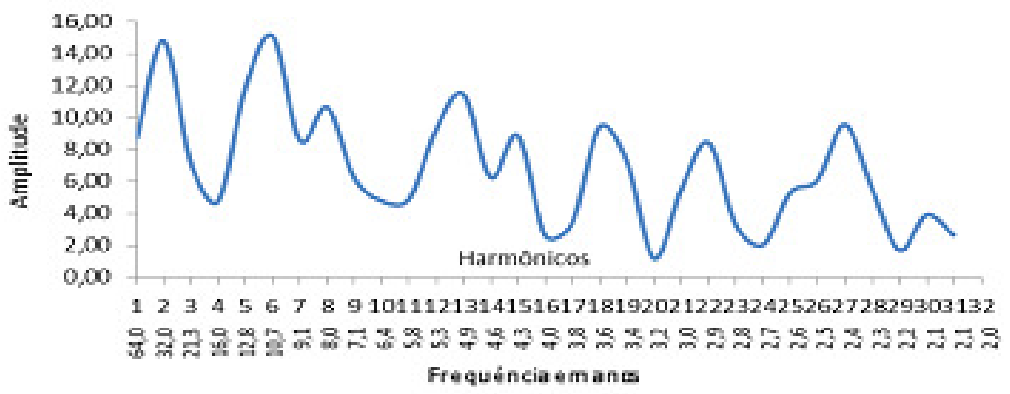

Figura 5. Análise Harmônica do A- $\mathrm{I}_{\mathrm{a}(\mathrm{UNEP})}$ e B- $\mathrm{I}_{\mathrm{m}}$ para o município de Paracurú. Fonte: Corrêa, I.C.P. (2016). 


\section{Conclusão}

Conclui-se que para o Crato e Paracurú, as principais forçantes responsáveis pela variabilidade do clima, aplicando a análise harmônica, são os fenômenos El Niño/La Niña, juntamente com a zona de convergência intertropical. Entretanto, eventos como os vórtices ciclônicos dos altos níveis troposféricos também são responsáveis pela variabilidade interanual.

\section{Referências}

BUDYKO, M. I. 1958. The heat balance of the earth's surface. Washington: U.S. Department of Commerce. 258p. il.

CAVALCANTI, E. P.; SILVA, E. D. V. 1994. Estimativa da temperatura do ar em função das coordenadas locais. VIII Congresso Brasileiro de Meteorologia e II Congresso Latino-Americano e Ibérico de Meteorologia. Sociedade Brasileira de Meteorologia. Belo Horizonte, pp.154-157.

CAVALCANTI, E. P.; SILVA, V. P. R.; SOUSA, F. A. S. 2006. Programa computacional para a estimativa da temperatura do ar para a região Nordeste do Brasil. Revista Brasileira de Engenharia Agrícola e Ambiental, v. 10, n. 1, p. 140-147.

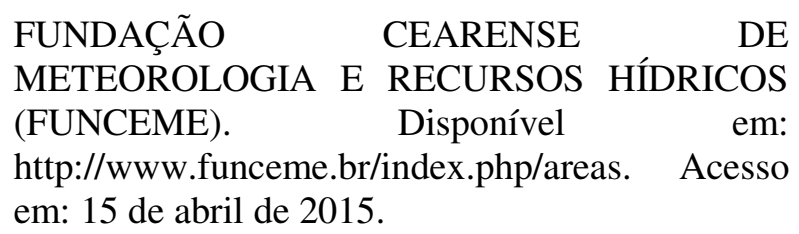

INSTITUTO BRASILEIRO DE GEOGRAFIA E ESTATÍSTICA. (IBGE). Disponível em: http://www.ibge.gov.br. Acesso em: 15 de abril de 2015.

INSTITUTO DE PESQUISA E ESTRATÉGIA ECONÔMICA DO CEARÁ (IPECE). 2007. Disponível em: http://www2.ipece.ce.gov.br/atlas/capitulo1/11/11 1x.htm. Acesso em: 15 de abril de 2015.

\begin{abstract}
INSTITUTO NACIONAL
DE METEOROLOGIA (INMET). Normais climatológicas 1961-1990. Brasília: INMET, 1997. Disponível em: http://www.inmet.gov.br/ Acesso em: 10 de julho de 2015.
\end{abstract}

NAKKEN, M. 1999. Wavelet analysis of rainfallrunoff variability isolating climatic from anthropogenic patterns. Environmental Modelling and Software, v. 14, p. 283-295.

ROLIM, G. S.; SENTELHAS, P. C. 1999. Planilhas no ambiente EXCEL para os cálculos de balanços hídricos: normal, sequencial, de cultura e de produtividade real e potencial. Revista Brasileira de Agrometeorologia, v. 6, p. 133-137.

SILVA, V.; SOUSA, F.; CAVALCANTI, E. P.; SOUZA, E.; DA SILVA, B. B. 2006. Teleconnections between sea-surface temperature anomalies and air temperature in northeast Brazil. Journal of Atmospheric and Solar-Terrestrial Physics, v. 68, n. 68, p. 781-792.

THORNTHWAITE, C. W. 1948. An approach toward a rational classification of climate. Geography Review, v. 38, p. 55-94.

THORNTHWAITE, C. W.; MATHER, J. R. 1955. The water balance: publications in climatology. New Jersey: Drexel Institute of Technology. 104p.

UVO, C. B. 1989. A zona de convergência intertropical (ZCIT) e sua relação com a precipitação na região norte do nordeste brasileiro. Dissertação de Mestrado. INPE, São José dos Campos. 99p.

TORRENCE, C.; COMPO, G. P. 1998. A Practical Guide to Wavelet Analysis. Bulletin of the American Meteorological Society, v. 79, p. 61-78.

UNITED NATIONS ENVIRONMENT PROGRAMME (UNEP). 1992. World Atlas of Desertification. London: UNEP/Edward Arnold, $69 \mathrm{p}$. 